For this axis, one-half the spacing in magnetic field between the $\left(\frac{1}{2} \leftrightarrow \frac{1}{2}\right)$ and $\left(+\frac{1}{2} \leftrightarrow+\frac{3}{2}\right)$ lines, which is an effective $|D|$ value for this axis to second order is $2390 \mathrm{G}$ for $\mathrm{Fe}^{3+}$ in thortveitite, $1103 \mathrm{G}$ for $\mathrm{Mn}^{2+}$ in $\mathrm{Zn}_{2} \mathrm{P}_{2} \mathrm{O}_{7}$ and $1180 \mathrm{G}$ for $\mathrm{Mn}^{2+}$ in $\mathrm{Mg}_{2} \mathrm{P}_{2} \mathrm{O}_{7}$. Since the last value becomes $1814 \mathrm{G}$ in the low-temperature phase where, in general, there are only modest structure changes, it is not inconceivable that the thortveitite structure gives a large axial field at the paramagnetic center. The low value of $E$ is again in keeping with the results found for the pyrophosphate analogs of thortveitite.
The data taken do not permit one to speculate as to either the site or the mechanism for charge compensation of $\mathrm{Mn}^{2+} \mathrm{Sc}_{2} \mathrm{Si}_{2} \mathrm{O}_{7}$. It would probably be advisable to prepare synthetic crystals to obtain a resolution.

\section{ACKNOWLEDGMENTS}

We wish to thank Dr. J. W. Adams and Dr. G. F. Claringbull for supplying samples of thortveitite and Dr. D. S. Russel of the National Research Council for chemical analysis of samples. Financial support of the research by the Ontario Department of University Affairs is acknowledged.

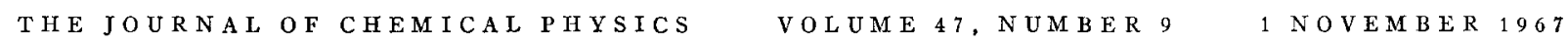

\title{
Benzene Vibrational Exciton Spectrum
}

\author{
RAOUL KOPELMAN \\ Department of Chemistry, California Institute of Technology, Pasadena, California* and Department of Chemistry, \\ University of Michigan, Ann Arbor, Michigan $\dagger$
}

(Received 15 June 1967)

\begin{abstract}
A critical discussion of the infrared polarization assignments of Zwerdling and Halford, in view of the now accepted benzene crystal structure, leads to acceptance of their results, though with somewhat reduced credibility. The controversial $707-\mathrm{cm}^{-1}$ absorption is assigned as the $B_{2}$ interchange component (b axis polarized) of the $a_{2 u}$ fundamental $\left(674-\mathrm{cm}^{-1}\right.$ gas-phase $)$ exciton band. The resulting, unusually large, static and dynamic exciton interaction terms are tabulated. Recent calculations based on atom-atom interactions are in reasonable agreement with the above results.
\end{abstract}

\section{INTRODUCTION}

There has recently been a revival of interest in both the electronic and vibrational exciton bands of benzene. ${ }^{1,2}$ In spite of both theoretical and experimental advances ${ }^{1,2}$ one still seems to depend on old experimental polarization data $^{3}$ and their interpretation. The latter was based on an old and incorrect crystal structure of benzene. This note attempts to analyze critically the available information (ir) and come to a tentative conclusion as to the "most likely assignment" of factorgroup ("Davydov") components. Without such an assignment one cannot uniquely correlate the spectroscopic interaction (coupling) terms with specific pairs of molecules in the unit cell. The conclusion arrived at is that the polarization assignments by Zwerdling and Halford ${ }^{3}$ are most probably correct for the fundamentals and other intense (ir) vibrational exciton

\footnotetext{
* Contribution No. 3537 .

$\dagger$ Present address.

1 (a) E. R. Bernstein, S. D. Colson, R. Kopelman, and G. W. Robinson (unpublished data). (b) Proc. Intern. Symp. Mol. Struct. Spectry., Columbus, Ohio, 1965, Paper M2.

${ }^{2}$ (a) S. D. Colson, R. Kopelman, and G. W. Robinson, J. Chem. Phys. 47, 27, (1967). (b) R. Gee, J. Chem. Phys. 46, 4847 (1967). (c) R. Kopelman, "The Interchange Symmetry 1: Molecules and Crystals", J. Chem. Phys. 47, 2631 (1967).

${ }^{3}$ S. Zwerdling and R. S. Halford, J. Chem. Phys. 23, 2221 (1955).
}

bands. Some experimental $a_{2 u}\left(\nu_{11}\right)$ interaction terms are derived and compared with recent calculations.

\section{THE POLARIZATIONS}

The pioneering works of Halford, Hornig, and their collaborators ${ }^{4}$ on benzene laid down the experimental foundations of vibrational spectra in molecular crystals. Mair and Hornig ${ }^{4 b}$ completed the vibrational assignment of the benzene molecule and discovered a number of crystal splittings. Zwerdling and Halford ${ }^{3}$ (henceforth $\mathrm{ZH}$ ) obtained polarized spectra of the single crystal. They gave a self-consistent account of all observed fine structure and therefrom identified the crystallographic directions of their specimen. Unfortunately, $\mathrm{ZH}$ relied on the old crystal structure of Cox, ${ }^{5}$ giving the molecular planes as parallel to the crystallographic b axis. As a result, $\mathrm{ZH}$ predicted certain factor-group components to be of vanishing intensity. ${ }^{6}$ Therefrom

4 (a) R. S. Halford and O. H. Schafer, J. Chem. Phys. 14, 141 (1946) (b) R. D. Mair and D. F. Hornig, J. Chem. Phys. 17, 1236 (1949).

${ }^{5}$ E. G. Cox, Proc. Roy. Soc. (London) Al35, 491 (1932).

${ }^{6} \mathrm{ZH}$ stressed, correctly, that this conclusion was derived from the "accidental" structure (which turned out to be wrong), and not from selection rules based on the space group (which are correctly stated in their paper and are still applicable because only the structure, but not the space group, of benzene has heen modified since - Ref. 7). 
Table I. Exciton terms for the $a_{2 u}\left(\nu_{11}\right)$ band $\left(\mathrm{cm}^{-1}\right)$.

\begin{tabular}{|c|c|c|c|c|c|c|c|}
\hline & $M_{\mathrm{III}}$ & $M_{\text {I III }}$ & $M_{\text {I IV }}$ & $2\left(M_{a}+M_{b}+M_{c}\right)$ & $(P+D)$ & {$\left[P+D+2\left(M_{a}+M_{b}+M_{c}\right)\right]$} & $\beta$ \\
\hline Experimental I & 1.2 & 4.6 & 1.4 & $2^{b}$ & 23 & 25 & 5 \\
\hline Experimental II & 0.7 & 4.1 & 0.9 & $0^{\mathrm{b}}$ & 23 & 23 & 4 \\
\hline Experimental III & 0.2 & 3.6 & 0.4 & $-2^{b}$ & 23 & 21 & 4 \\
\hline Experimental IV & -0.3 & 3.1 & -0.1 & $-4^{\mathrm{b}}$ & 23 & 19 & 3 \\
\hline Calculated & -0.1 & 2.7 & 0.2 & $?$ & $?$ & 21 & $3^{\mathrm{d}}$ \\
\hline
\end{tabular}

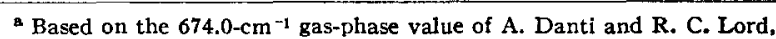
Spectrochim. Acta 13, 180 (1958).

b Assumed ("parameter"), see text.

$\mathrm{ZH}$ derived the clear-cut conclusion that their specimen had its $\mathbf{b}$ axis along the direction of radiation propagation. In view of the now-accepted crystal structure $^{7}$ this conclusion can no longer be justified by the original arguments. However, the fact remains that some band components observed previously by Mair and Hornigb (and many times since, including recent work ${ }^{8}$ ) were not observed by $\mathrm{ZH}$, even though their instrumental setup seemed capable of observing them. Therefore, remembering that the space group ( $P b c a$ ) is orthorhombic we come here to the definite conclusion that one major crystallographic axis was oriented along the radiation propagation direction in the $\mathrm{ZH}$ experiment. On the other hand, $\mathrm{ZH}$ used the oriented gas model to identify their $\mathbf{a}$ and $\mathbf{c}$ axes. ${ }^{9}$ The dichroic ratio expected between a- and $\mathbf{c}$-axis-polarized absorptions, based on the oriented gas model, does not change much from the old to the new crystal structure. Therefore, the same criterion would still lead to the identifcation of the two observed axes as a and $\mathbf{c}$ (as quoted by $\mathrm{ZH}$ ) while the $\mathbf{b}$ axis would be assigned again as the one parallel to the radiation propagation direction. ${ }^{10}$

Some additional support to the above identification of axes can be derived from the hitherto most controversial feature of the benzene-crystal ir spectrum: the $a_{2 u}$ fundamental at $680 \mathrm{~cm}^{-1}$, or more specific, its weak component around $705 \mathrm{~cm}^{-1}$. Mair and Hornig,tb as well as $\mathrm{ZH}$, did not formally assign the latter (weak) absorption. It is polarized according to $\mathrm{ZH}$ mainly along the $\mathbf{c}$ axis, but seems to have a definite component along a, while the $680-\mathrm{cm}^{-1}$ (very intense) absorption is almost equally intense along $\mathbf{a}$ and $\mathbf{c}$ directions with the a polarized intensity being slightly

${ }^{7}$ E. G. Cox Rev. Mod. Phys. 10, 159 (1958); E. G. Cox, J. W. J. Cruichshank, and J. A. S. Smith, Proc. Roy. Soc. (London) A247, 1 (1958); G. E. Bacon, N. A. Curry, and S. A. Wilson, Proc. Roy. Soc. (London) A279, 98 (1964).

${ }_{8}^{8} \mathrm{~J}$. L. Hollenberg and D. A. Dows, J. Chem. Phys. 37, 1300 (1962). Also E. Bernstein (private communication).

${ }^{9}$ Wisely, they took as their criterion for this identification only the non-crystal-induced bands. In view of later evidence (Ref. 8) that the intensity of these bands changes little upon condensation, it may still be a valid criterion.

${ }^{10}$ However, only because the dichroic ratio $c / a$ is expected, for intrinsically allowed fundamentals, to be close to unity (oriented gas model) while the ratio $b / a$ or $b / c$ is expected to be much smaller. For instance, for an out-of-plane $\left(A_{2 u}\right)$ band, $a / c \cong 1.1$, $b / a \cong 0.1$. This seems to lend much more weight to ZH's identification of the $a c$ plane than to their specific identification of the $a$ and $c$ axes.
Derived from Ref. 24 .
d Assuming $2\left(M_{a}+M_{b}+M_{c}\right)$ is small. This may not be so.

greater than the c polarized intensity. Hexter and Dows ${ }^{11}$ assigned the $705-\mathrm{cm}^{-1}$ components as a combination band of the $a_{2 u}$ fundamental and a rotational lattice mode of about $17 \mathrm{~cm}^{-1}$ (supposedly not resolved in Raman spectra ${ }^{12}$ ). Cruickshank ${ }^{13}$ argued that such a lattice mode does not fit vibration amplitudes derived from $x$-ray investigations, nor the experimental entropy vs temperature curve of the crystal. Person and Olsen ${ }^{14}$ argued that the 704 absorption is a member of the factor-group structure ${ }^{15}$ but did not specify which one. Hollenberg and Dows ${ }^{16}$ made more refined measurements, (higher resolution, 5\% mole mixed crystal). The peak apparently pertaining to "isolated" $\mathrm{C}_{6} \mathrm{H}_{6}$ in

${ }_{11}^{11}$ R. M. Hexter and D. A. Dows, J. Chem. Phys. 25, 504 (1956). A suggestion in this direction is found in Ref. 3, too.

${ }^{12}$ S. C. Sirkar and A. K. Ray, Ind. J. Phys. 24, 189 (1950). S. C. Sirkar, D. K. Mukherjee, and P. K. Bishue, Ind. J. Phys. 38, 181 (1964). M. Ito, J. Chem. Phys. 42, 2844 (1965). The claim made in this work that the site selection rules are violated is unjustified in our opinion. The $1012-\mathrm{cm}^{-1}$ band can be easily interpreted as the $b_{2 \sigma}$ fundamental, $\nu_{5}$. It is observed ${ }^{12 a}$ at 1004 $\mathrm{cm}^{1}$ in a mixed crystal. The relatively large shift from the liquid value of $995 \mathrm{~cm}^{-1}$ (if indeed 995 is correctly assigned as $\nu_{5} !$ ) could be explained as due to Fermi resonance of $\nu_{3}$ with the totally symmetric $\nu_{1}\left(992 \mathrm{~cm}^{-1}\right)$, as in the site $C_{i}$ they have the same symmetry $\left(A_{0}\right)$. Very recently a similar conclusion has been reached independently by $M$. Ito and $T$. Shigeoka, Spectrochim. Acta 22, $1029(1966)$. The difference between the liquid value $\left(995 \mathrm{~cm}^{-1}\right)$ and the solid value may also be partly due to crystal splittings, not shift. Such splittings [see Ref. 2(b)] could also account for the difference between the solid value inferred from ir combinations and the one observed in the Raman, assuming that not all interchange (factor) group components have been observed in the Raman spectrum.

12a G. C. Nieman, Ph.D. thesis, California Institute of Technology (1964).

${ }^{13}$ D. W. J. Cruickshank, Rev. Mod. Phys. 30, 163 (1958). ${ }^{14} \mathrm{~W}$. B. Person and D. A. Olsen, J. Chem. Phys. 32, 1268 (1960).

${ }^{16}$ Their conclusion is based on the observation that in a $10 \%$ mole solid solution of $\mathrm{C}_{6} \mathrm{H}_{6}$ in $\mathrm{C}_{6} \mathrm{D}_{6}$, the absorption moved to a value halfway between 690 and $705 \mathrm{~cm}^{-1}$. In a fourfold factorgroup structure, as that of benzene, such a "half-way" requirement is neither a necessary result of, nor a sufficient condition for, such an assionment. Even in a twofold factor-group structure such a "half-way" absorption is not a necessary result, due to the "translational (Ref. 1) shift" (and also quasiresonance). However, the observed drastic shift $\left(15 \mathrm{~cm}^{-1}\right)$ upon dilution (a $10 \%$ mole solution certainly does not isolate the solute statistically) does indicate a wide "exciton band" and thus, indirectly, argues for the inclusion of the $705-\mathrm{cm}^{-1}$ absorption in the multiplet structure of the $680-\mathrm{cm}^{-1}$ hand. Another good argument is the analogous behavior of the spectra of $\mathrm{C}_{6} \mathrm{D}_{6}$ and of $\mathrm{C}_{6} \mathrm{D}_{6}$ diluted in $\mathrm{C}_{8} \mathrm{H}_{6}$. Their term "correlation doubling" should not be taken literally.

${ }^{16} \mathrm{~J}$. L. Hollenberg and D. A. Dows, J. Chem. Phys. 39, 495 (1963). 
$\mathrm{C}_{6} \mathrm{D}_{6}$ is at $697.3 \mathrm{~cm}^{-1}$; that of "isolated" $\mathrm{C}_{6} \mathrm{D}_{6}$ in $\mathrm{C}_{6} \mathrm{H}_{6}$ at $511.6 \mathrm{~cm}^{-1}$. The frequencies of the pure crystals are ${ }^{8}$ : 679.7, 681.4, 706.6 $\mathrm{cm}^{-1}$ for $\mathrm{C}_{6} \mathrm{H}_{6}$, and 502.1 and $519.0 \mathrm{~cm}^{-1}$ for $\mathrm{C}_{6} \mathrm{D}_{6}$. (The 527.9- $\mathrm{cm}^{-1}$ component does not seem to belong to the multiplet. ${ }^{16}$ ) The question is: How can the third factor-group component be observed (by $\mathrm{ZH}$ ) along the same two axes (a and $\mathbf{c}$ ) as the other two components? The following answer is suggested: The observation is due to an imperfection in the experimental setup of $\mathrm{ZH}$, and can be explained, quantitatively, as follows: The light traversing the sample is not exactly parallel light. A schematic representation of the setup reveals that the edge of the beam is at an angle of about $2.5 / 30$ radians off-parallel. This can introduce the $\mathbf{b}$ axis component with a relative average intensity of about ${ }^{17}\left(\frac{1}{2} 2.5 / 30 n\right)^{2} \cong 0.001$. The total intensity of the $\mathbf{b}$ component (assumed now to be assigned as the $706.6-\mathrm{cm}^{-1}$ absorption) is about $6 \%$ of the total of the three components. ${ }^{18}$ (The oriented gas model happens to predict $5.0 \%$.) The total intensity of the $a_{2 u}$ band is about 9200 darks. $^{8}$ (It is the most intense band of the system!) Thus, the component in question is expected to appear with an intensity of about one dark in the $\mathrm{ZH}$ spectrum. ${ }^{19}$ The intensity of the weakest (induced) fundamental, $\nu_{14}$, is only about 34 darks. ${ }^{8}$ So the $\mathbf{b}$ component of the most intense band is indeed expected to appear with about a few percent of the intensity of the $\nu_{14}$ fundamental. ${ }^{20}$ It may be pointed out that the other fundamentals are all much weaker ${ }^{16}$ than the $a_{2 u}$ and therefore their $b$-axis components are not expected to be revealed by the $\mathrm{ZH}$ spectra (with the possible exception of $\nu_{19}$ ). This rather involved, but quite satisfactory, explanation of the $705-\mathrm{cm}^{-1}$ component and its appearance in the spectra recorded by $\mathrm{ZH}$ rested on the assumption that in their experiment the $\mathbf{b}$ axis was indeed parallel (or very nearly so) to their "propagation direction," or, actually, to the short axis of their cell. Actually, being able to develop such a consistent explanation seems to support strongly the b axis assignment, ${ }^{21}$ and thereby our acceptance of the $\mathrm{ZH}$ identification of axes.

\footnotetext{
${ }^{17}$ Here $n$ is the index of refraction. A helpful comment by $\mathrm{I}$. Freund is gratefully acknowledged.

${ }^{18}$ Measured roughly from the spectra of Ref. 16.

${ }_{19} 0.001 \times 0.06 \times 9200=0.6$. As the $c$ axis makes a $30^{\circ}$ angle with the "vertical" (the slit direction), the " $c$ " polarization spectrum should reveal the $b$ component more intensely than the " $a$ " polarization. This seems indeed to be the case. Obviously, an additional misalignment between the $b$ axis and the center-ofbeam axis cannot be ruled out.

${ }^{20}$ Indeed, the $680-\mathrm{cm}^{-1}$ component ( 9000 darks) is given as "9," that of the 24 darks $\nu_{14}$ is given as " 6 ," that of $705 \mathrm{~cm}^{-1}$ as " 3 " by ZH. (These are very rough intensity designations.)

21 The only reasonable alternative is to account for the $705-\mathrm{cm}^{-1}$ peak as an "artifact" (impurity, imperfection). Such an "artifact" level may find itself inside the $a_{t u}$ exciton band and may, therefore, be able to "borrow" from its intensity-see E. I. Rashba. Soviet Phys. - Solid state 4, 2417 (1963) [Fiz. Tverd. Tela 4, 3301 (1962) ]. With such an alternative one is tempted to assign the b component to the shoulder discernible in Hollenberg and Dows 816 spectrum at about $690 \mathrm{~cm}^{-1}$. Such alternative assignment would not affect drastically the $M$ terms calculated in the next paragraph.
}

It may be stressed again that all of the above discussion rested on the validity of the oriented gas model. While we cannot expect, in general, quantitative results from this model we may accept it on a qualitative, or semiquantitative basis, for bands that change little in intensity from gas to crystal. Obviously, once the polarization directions have been defined in an orthorhombic crystal for one band they have been defined for all of them.

\section{THE $a_{2 u}$ FUNDAMENTAL}

We venture ${ }^{22}$ to assign the $\mathrm{ZH}$ a- and c-polarized components ( 688 and $689 \mathrm{~cm}^{-1}$ ) of the $a_{2 u}$ fundamental to the Hollenberg and Dows ${ }^{8} 679.7-$ and $681.4-\mathrm{cm}^{-1}$ components, respectively (a large calibration error in this difficult region of the $\mathrm{NaCl}$ prism is quite possible). Assuming a positive translational shift, ${ }^{1}$ $2\left(M_{a}+M_{b}+M_{c}\right)$, of about $2 \mathrm{~cm}^{-1}$ (by analogy ${ }^{1}$ with $\left.{ }^{1} B_{2 u}\right)$ and with a quasiresonance shift of $-0.5 \mathrm{~cm}^{-1}$ (by iteration) ${ }^{1}$ we get the mean of the band at 699 $\mathrm{cm}^{-1}$. The inactive factor-group component is therefore at $728.3 \mathrm{~cm}^{-1}$. The total splitting is about $50 \mathrm{~cm}^{-1}$ ! This large splitting seems to correlate with the fact that $a_{2 u}$ is, by far, the strongest fundamental band (its intensity is larger than the combined intensity of all the others $\left.{ }^{8}\right)$. However, an elementary calculation shows that any contribution from dipole-dipole interactions is too small to account for it by an order of magnitude. Also, the site shift ${ }^{1}(P+D)$ is very large: $+23 \mathrm{~cm}^{-1}$. Individual and average exciton interaction terms ${ }^{1}$ are given in Table I with the translational shift as "parameter." These terms (especially $M_{\mathrm{I} \text { III }}$ and $\beta$ ) are much larger than such interaction terms of other vibrations, and seem to approach the values of the electronic exciton bands. ${ }^{1,23}$

It may be worthwhile to compare the experimental values (Table I) with those calculated recently ${ }^{24}$ by the semiemperical atom-atom model. While the agreement is not perfect (Table I), it is surprising to find it as good as it is. Note that this agreement becomes even better if we assume a negative instead of a positive translational shift. It is also of interest to compare the large gas-to-crystal $(P+D)$ shift of $23 \mathrm{~cm}^{-1}$ with the small gas-to-liquid shift $\left(\sim 1 \mathrm{~cm}^{-1}\right){ }^{25}$

Analogous treatments, using the polarization assignments as given above, have been applied to two other

\footnotetext{
${ }^{22}$ A preliminary discussion was given - Ref. 1(b).

${ }_{23} \mathrm{It}$ should be pointed out that $\beta$ of Table $\mathrm{I}$ is of the same order as that of the ${ }^{1} B_{2 u}$ electronic state (Ref. 1) and the ${ }^{3} B_{1 u}$ electronic state: G. C. Nieman and G. W. Robenson, J. Chem. Phys. 39, 1298 (1963).

${ }^{24}$ I. Harada and T. Shimanouchi, J. Chem. Phys. 44, 2016 (1966). Similar conclusions have been drawn by N. H. Rich and D. A. Dows, Proc. Intern. Symp. Mol. Struct. Spectry., Columbus, Ohio, 1965. Paper V11.

${ }_{25}$ The literature value [i.e., Ref. 4(b)] for the liquid, at room temperature. $675 \mathrm{~cm}^{-1}$, may not be very accurate (due to calibration problems in this region, $\mathrm{CO}_{2}$, and the intensity of the band), but is still significantly lower then the lowest crystal bands, especially when taken under similar experimental conditions and at a comparable temperature of $-12^{\circ} \mathrm{C}$ [Ref. $\left.4(\mathrm{~b})\right]$.
} 
nondegenerate fundamentals very recently. ${ }^{1}$ The analysis of degenerate bands would be more complex, ${ }^{1,26}$ even if the polarizations of all six components were known.

Finally, could the wide exciton band of $a_{2 u}$, as well as those of other out-of-plane fundamentals, be related to the large out-of-plane amplitudes ${ }^{27}$ of vibration? This could be the case, qualitatively, with any intermolecular potential function. It remains to be seen how sensitive vibrational exciton bands are to such specific potentials.

\section{CONCLUSIONS}

The above analysis of the $a_{2 u}$ fundamental exciton band gives one further confidence in the polarization assignments of $\mathrm{ZH}$ for this band and hence for most other bands. Some of these assignments have already been utilized. ${ }^{1}$ Others may be utilized by future workers. We therefore feel that the polarization assignments of $\mathrm{ZH}$, though not all the arguments on which they were based, should be accepted, at least tentatively and until better evidence is made available.

The exciton ("factor-group") structure of the $a_{2 u}\left(\nu_{11}\right)$ fundamental seems to be finally settled, in a way consistent with all experimental observations. The exciton interaction terms are unusually large for vibrational bands of nonpolar molecular crystals. However, recent calculations, ${ }^{24}$ based on empirical atom-atom $(\mathrm{H}-\mathrm{H}$ only) interactions, are in surprisingly good agreement with the experimental conclusions.

Notes added in proof: (a) From a very recent paper, providing new data on benzene lattice modes [I. Harada and T. Shimanouchi, J. Chem. Phys. 46, 2708 (1967)], it becomes clear beyond any doubt that the $707-\mathrm{cm}^{-1}$ absorption is not related to lattice modes. (b) In a new investigation of single crystal spectra (A. Lubezky, M. Sc. thesis, Technion-Israel Institute of Technology, 1967) the three components of $\nu_{11}$ are given as 680,688 , and $708 \mathrm{~cm}^{-1}$ (tentatively assigned as the $\mathbf{a}, \mathbf{c}$, and $\mathbf{b}$ components, respectively). In an

\footnotetext{
${ }^{26}$ R. Kopelman, J. Chem. Phys. 44, 3547 (1966).
}

27 (a) W. V. F. Brooks, S. J. Cyvin, and P. C. Kvande, J. Phys. Chem. 69, 1489 (1965). (b) W. V. F. Brooks and S. J. Cyvin, Spectrochim. Acta 18, 397 (1962). (c) W. V. F. Brooks and S. J. Cyvin, Acta Chem. Scand. 16, 820 (1962). See also: A. C. Albrecht, J. Mol. Spectry. 5, 236 (1960). A quantitative comparison of the various normal mode amplitudes and the experimentally known vibrational exciton bands seems to be premature at this point. isolated experiment the $708-\mathrm{cm}^{-1}$ component was found to be "polarized." This is in general agreement with the present paper, but the new experimental evidence does not seem to be conclusive. (c) An earlier manuscript came to the author's attention (L. C. Hall, Ph.D. thesis, University of Iowa, 1961). It has already been suggested there that the "705" component should be assigned as the transition along the $\mathbf{b}$ crystal axis. Hall investigated also mixed crystals $\left(\mathrm{C}_{6} \mathrm{H}_{6}-\mathrm{C}_{6} \mathrm{D}_{6}\right)$. At medium low concentrations of $\mathrm{C}_{6} \mathrm{H}_{6}$ a band at 693.5 $\mathrm{cm}^{-1}$ seems to persist, while at low concentration only the $697.5 \mathrm{~cm}^{-1}$ band is clearly evident. I tentatively assign the $693.5 \mathrm{~cm}^{-1}$ feature as a "resonance pair" ("dimer") line (the $697.5-\mathrm{cm}^{-1}$ one being the "monomer" absorption). From Table I we assume $M_{\text {I III }}$ to be the largest pairwise interaction term, and therefore assign the 693.5 feature as the I-III pair "out of phase" (using a $\mathrm{D}_{2}$ interchange convention ${ }^{1,2}$ ) state. Note that the molecular transition moments are nearly parallel in this state. The "in-phase" $\left(\mathbf{D}_{2}\right.$ convention) state transition moment is expected to be weak (molecular moments nearly antiparallel). The I-II and I-IV resonance-pair states are expected to be close enough to the monomer state so as not to be resolved. Assuming the resonance-pair ("dimer") states to be about evenly split with respect to the mononer "center" state, one gets directly the pairwise interaction term $M_{\text {I III }} \cong 4 \mathrm{~cm}^{-1}$. This is in excellent agreement with the value (and sign!) derived from the pure crystal, $3.8 \pm 0.8 \mathrm{~cm}^{-1}$ (see Table I). It is therefore also consistent with the idea that nearest neighbor interactions dominate (i.e. a steep inverse dependence on distance). The above is also consistent with a small translation shift $\left(M_{a}+M_{b}+M_{c}\right)$, making "Experimental II" (Table I) the most probable set of results (including $M_{\mathrm{I} \text { III }}=4.1 \mathrm{~cm}^{-1}$ !) . Finally, noting that the I-III pair consists of nearly parallel molecules, while the I-II and I-IV pairs consist of nearly perpendicular molecules, one can in this way rationalize the fact that $M_{\text {I III }}$ is quite different from $M_{\text {I II }}$ and $M_{\text {I IV }}$, without having to assume a specific interaction model.

\section{ACKNOWLEDGMENT}

The author would like to thank E. R. Bernstein, S. D. Colson, G. W. Robinson, R. S. Halford, and Isaac Freund for helpful comments. Correspondence with D. A. Dows and W. B. Person is also acknowledged. 